\title{
Scattering Theory for Elastic Wave Propagation Problems in Perturbed Stratified Media II
}

By

\author{
Senjo SHIMIzU*
}

\begin{abstract}
We consider a self-adjoint operator governing the propagation of elastic waves in stratified media $\boldsymbol{R}^{3}$, where Lamé functions and a density are perturbed in a compact region. In this paper we prove the existence, the completeness, and the invariance principle of wave operators associated with the self-adjoint operator and a self-adjoint operator governing the propagation of elastic waves in unperturbed stratified media $\boldsymbol{R}^{3}$. The proof is based on an abstract scattering theory due to M. $\breve{S}$. Birman.
\end{abstract}

\section{§1. Introduction and Results}

In this paper we consider propagation problems of elastic waves in perturbed stratified media $\boldsymbol{R}^{3}$ with free boundary-interface conditions.

The purpose of this paper is to prove the existence, the completeness, and the invariant principle of the wave operators associated with self-adjoint operators for elastic wave propagation problems in stratified media $\boldsymbol{R}^{3}$ using methods due to Birman. In the previous paper we proved the existence, the completeness, and the invariant principle for elastic wave propagation problems in the case where only Lamé functions are perturbed in a compact region [Sh 3]. In this paper we treat the more general case where both Lamé functions and a density are perturbed in a compact region. Wilcox's work [Wi] is useful reference to our study.

We start with a formulation of elastic wave propagation problems in perturbed stratified media $\boldsymbol{R}^{3}$.

Let $\Omega$ be an exterior domain in $\boldsymbol{R}^{3}=\left\{x=\left(x^{\prime}, x_{3}\right)=\left(x_{1}, x_{2}, x_{3}\right) \mid x_{i} \in \boldsymbol{R}\right\}$ of

Communicated by Y. Takahashi, May 13, 1996. Revised December 24, 1996.

1991 Mathematics Subject Classification: Primary 35P25, 47A55; Secondary 73C35.

This work is partially supported by the Grant-in-Aid for the Encouragement of Young Scientists A-08740097 from the Ministry of Education, Science and Culture of Japan.

* Faculty of Engineering, Shizuoka University, Hamamatsu 432, Japan.

E-mail address: tssshim@eng.shizuoka.ac.jp 
which boundary $\partial \Omega$ is smooth and compact. Let $\lambda(x)$ and $\mu(x)$ denote Lamé functions in $\Omega$, and $\rho(x)$ denote a density function in $\Omega$. We assume that there exist positive constants $m$ and $M$ such that

$$
0<m \leq \lambda(x), \mu(x), \rho(x) \leq M \quad \text { for a.e. } x \in \bar{\Omega},
$$

where (cf. Figure 1)

$$
\begin{aligned}
& \lambda(x)=\left\{\begin{array}{lll}
\lambda_{1}(x) & \text { for } & x \in \Omega \cap \boldsymbol{R}_{-}^{3}, \\
\lambda_{2}(x) & \text { for } & x \in \Omega \cap \mathbb{R}_{+}^{3},
\end{array}\right. \\
& \mu(x)=\left\{\begin{array}{lll}
\mu_{1}(x) & \text { for } & x \in \Omega \cap \boldsymbol{R}_{-}^{3}, \\
\mu_{2}(x) & \text { for } & x \in \Omega \cap \boldsymbol{R}_{+}^{3},
\end{array}\right. \\
& \rho(x)=\left\{\begin{array}{lll}
\rho_{1}(x) & \text { for } & x \in \Omega \cap \boldsymbol{R}_{-}^{3}, \\
\rho_{2}(x) & \text { for } & x \in \Omega \cap \boldsymbol{R}_{+}^{3},
\end{array}\right. \\
& \lambda_{1}(x), \mu_{1}(x), \rho_{1}(x) \in C^{1}\left(\Omega \cap \boldsymbol{R}_{-}^{3}\right), \\
& \lambda_{2}(x), \mu_{2}(x), \rho_{2}(x) \in C^{1}\left(\Omega \cap \boldsymbol{R}_{+}^{3}\right),
\end{aligned}
$$

and the functions $\lambda_{1}(x), \lambda_{2}(x), \mu_{1}(x), \mu_{2}(x), \rho_{1}(x), \rho_{2}(x)$ are constant for $|x|$ sufficiently large, that is, for a large constant $L>0$

$$
\begin{array}{lll}
\left(\lambda_{1}(x), \mu_{1}(x), \rho_{1}(x)\right)=\left(\lambda_{1}, \mu_{1}, \rho_{1}\right) & \text { for } & x \in \mathbb{R}_{-}^{3},|x|>L, \\
\left(\lambda_{2}(x), \mu_{2}(x), \rho_{2}(x)\right)=\left(\lambda_{2}, \mu_{2}, \rho_{2}\right) & \text { for } & x \in \mathbb{R}_{+}^{3},|x|>L .
\end{array}
$$

Here $\boldsymbol{R}_{-}^{3}=\left\{x \in \boldsymbol{R}^{3}, x_{3}<0\right\}$ and $\boldsymbol{R}_{+}^{3}=\left\{x \in \boldsymbol{R}^{3}, x_{3}>0\right\}$.

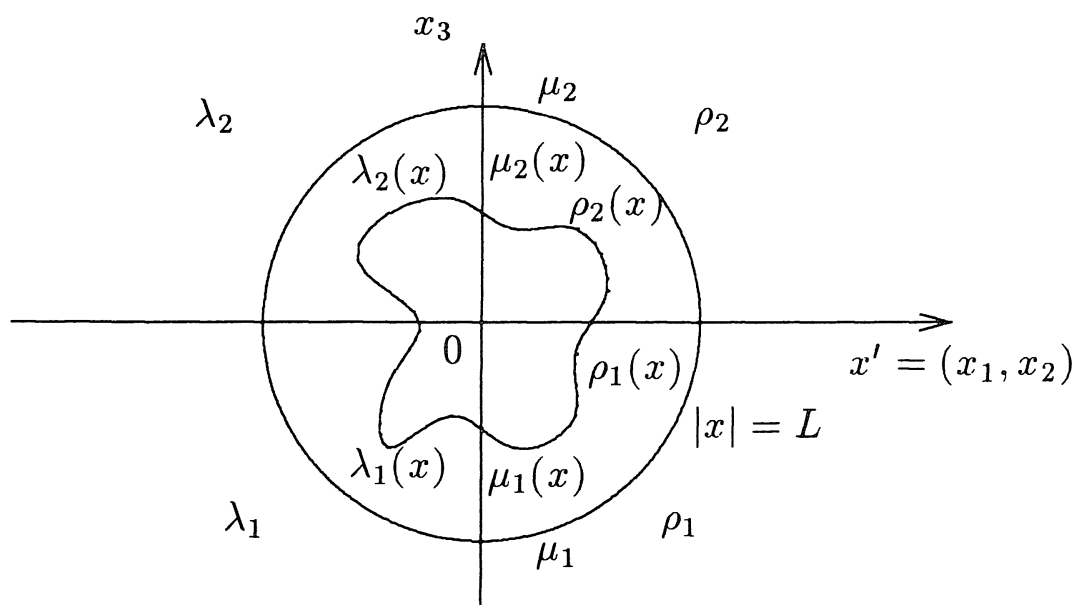

Figure 1. Perturbed Stratified Medium $\boldsymbol{R}^{3}$ 
Let $u(t, x)={ }^{t}\left(u_{1}(t, x), u_{2}(t, x), u_{3}(t, x)\right) \in \boldsymbol{R}^{3}$ be the displacement vector at time $t$ and position $x$. The propagation problem of elastic waves in the perturbed stratified medium $\boldsymbol{R}^{3}$ is formulated as the following mixed problem:

$$
\begin{aligned}
& \frac{\partial^{2} u_{k}}{\partial t^{2}}(t, x)-\frac{1}{\rho(x)} \sum_{j=1}^{3} \frac{\partial}{\partial x_{j}} \sigma_{k j}(u(t, x))=0, \quad x=\Omega, \\
& \left.u(t, x)\right|_{\Omega \cap\left\{x_{3}=-0\right\}}=\left.u(t, x)\right|_{\Omega \cap\left\{x_{3}=+0\right\}}, \\
& \left.\sigma_{k 3}(u(t, x))\right|_{\Omega \cap\left\{x_{3}=-0\right\}}=\left.\sigma_{k 3}(u(t, x))\right|_{\Omega \cap\left\{x_{3}=+0\right\}}, \\
& \left.\sum_{j=1}^{3} \sigma_{k j}(u(t, x)) v_{j}\right|_{\partial \Omega}=0 \\
& u(0, x)=f(x), \quad \frac{\partial u}{\partial t}(0, x)=g(x)
\end{aligned}
$$

where

$$
\sigma_{k j}(u)=\lambda(\cdot)(\nabla \cdot u) \delta_{k j}+2 \mu(\cdot) \varepsilon_{k j}(u), \quad k, j=1,2,3
$$

are symmetric stress tensors,

$$
\varepsilon_{k j}(u)=\frac{1}{2}\left(\frac{\partial u_{k}}{\partial x_{j}}+\frac{\partial u_{j}}{\partial x_{k}}\right), \quad k, j=1,2,3,
$$

are symmetric strain tensors, and $v=\left(v_{1}, v_{2}, v_{3}\right)$ denotes the exterior normal at a point $x \in \partial \Omega$. The conditions (1.5) and (1.6) are called free interface conditions, the condition (1.7) is a free boundary condition, and the condition (1.8) is an initial condition. Here the word 'free' means Neumann type, and these free interface and boundary conditions appear in practical situations.

A Hilbert space and a self-adjoint operator are associated with solutions to the mixed problem (1.4)-(1.8) with finite energies in the following way: Let

$$
(\mathscr{A} u)_{k}=-\frac{1}{\rho(x)} \sum_{j=1}^{3} \frac{\partial}{\partial x_{j}} \sigma_{k j}(u)
$$

The Sobolev spaces on $\Omega$ are defined by

$$
\begin{array}{r}
H^{m}\left(\Omega, C^{3}\right)=\left\{u \in C^{3} \mid D^{\alpha} u \in L^{2}\left(\Omega, C^{3}\right) \text { for }|\alpha| \leq m\right. \\
\text { in the sense of distributions }\},
\end{array}
$$

where $m$ is a non-negative integer and the usual multi-index notation is used 
for derivatives. $H^{m}\left(\Omega, C^{3}\right)$ is a Hilbert space with the inner product

$$
(u, v)_{m}=\int_{\Omega} \sum_{|\alpha| \leq m} D^{\alpha} u(x) \cdot \overline{D^{\alpha} v(x)} d x,
$$

where $u \cdot \bar{v}$ denotes the usual scalar product in $C^{3}: u \cdot \bar{v}=\Sigma_{k=1}^{3} u_{k} \overline{v_{k}}$.

Definition 1.1. A function $u \in H^{1}\left(\Omega, C^{3}\right)$ satisfying $A u \in L^{2}\left(\Omega, C^{3}\right)$ is said to satisfy the generalized free boundary-interface condition if it satisfies the condition

$$
\sum_{k=1}^{3} \int_{\Omega}(\mathscr{A} u)_{k} \overline{\bar{v}_{k}} \rho(x) d x-\int_{\Omega}\left(\lambda(x)(\nabla \cdot u)(\nabla \cdot \bar{v})+\sum_{k, j=1}^{3} 2 \mu(x) \varepsilon_{k j}(u) \varepsilon_{k j}(\bar{v})\right) d x=0
$$

for every $v \in H^{1}\left(\Omega, C^{3}\right)$ (cf. [D-G]).

Even if the boundary of $\Omega$ is smooth, the boundaries of $\Omega \cap \boldsymbol{R}_{-}^{3}$ and of $\Omega \cap \boldsymbol{R}_{+}^{3}$ are not smooth. Therefore the interface conditions (1.5)-(1.6) and the boundary condition (1.7) would not be satisfied in the usual sense (cf. [Mi, Theorem 3.24]).

We introduce a Hilbert space

$$
\mathscr{H}=L^{2}\left(\Omega, C^{3}, \rho(x) d x\right)
$$

with the inner product

$$
(u, v)_{\mathscr{H}}=\int_{\Omega} u \cdot \bar{v} \rho(x) d x .
$$

Proposition 1.2. The operator $A$ in $\mathscr{H}$ with domain:

$$
\begin{aligned}
D(A)= & \left\{u \in H^{1}\left(\Omega, C^{3}\right) \mid u \text { satisfies } \mathscr{A} u \in L^{2}\left(\Omega, C^{3}\right)\right. \text { and } \\
& \text { the generalized free boundary-interface condition (1.14) }\}
\end{aligned}
$$

and action defined by

$$
A u=\mathscr{A} u, \quad u \in D(A)
$$

is a non-negative self-adjoint operator.

If we use Green's formula in the domains $\Omega \cap \boldsymbol{R}_{-}^{3}$ and $\Omega \cap \boldsymbol{R}_{+}^{3}$ separately, and use the generalized free boundary-interface condition (1.14), then we can 
prove Proposition 1.2 just as in [Sh 1, Theorem 1.2].

We consider the plane stratified medium $\boldsymbol{R}^{\mathbf{3}}$ with the planer interface $x_{3}=0$, which is defined by

$$
\left(\lambda_{0}\left(x_{3}\right), \mu_{0}\left(x_{3}\right), \rho_{0}\left(x_{3}\right)\right)=\left\{\begin{array}{lll}
\left(\lambda_{1}, \mu_{1}, \rho_{1}\right) & \text { for } & x_{3}<0 \\
\left(\lambda_{2}, \mu_{2}, \rho_{2}\right) & \text { for } & x_{3}>0
\end{array}\right.
$$

Here $\lambda_{1}, \lambda_{2}, \mu_{1}, \mu_{2}$, are Lamé constants and $\rho_{1}, \rho_{2}$ are constant densities (cf. Figure 2).

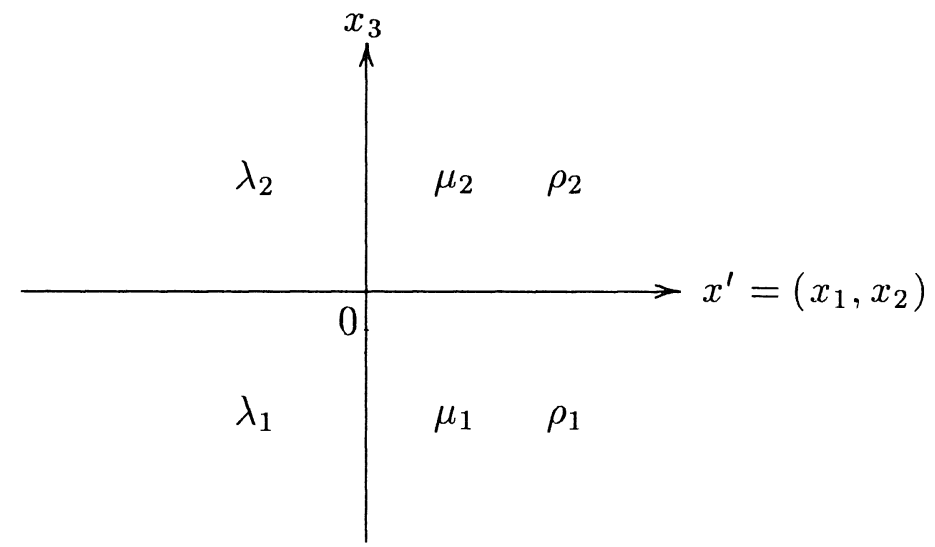

Figure 2. Unperturbed Stratified Medium $\boldsymbol{R}^{3}$

The propagation problem of elastic waves in this unperturbed stratified medium is formulated as the following initial-interface value problem:

$$
\frac{\partial^{2} u}{\partial t^{2}}(t, x)+\mathscr{A}_{0} u(t, x)=0
$$$$
\left.u(t, x)\right|_{x_{3}=-0}=\left.u(t, x)\right|_{x_{3}=+0},
$$

$$
\left.\sigma_{k 3}(u(t, x))\right|_{x_{3}=-0}=\left.\sigma_{k 3}(u(t, x))\right|_{x_{3}=+0}, \quad k=1,2,3,
$$$$
u(0, x)=f(x), \quad \frac{\partial u}{\partial t}(0, x)=g(x)
$$

where

$$
\mathscr{A}_{0} u=-\frac{\lambda_{0}\left(x_{3}\right)+\mu_{0}\left(x_{3}\right)}{\rho_{0}\left(x_{3}\right)} \nabla(\nabla \cdot u)-\frac{\mu_{0}\left(x_{3}\right)}{\rho_{0}\left(x_{3}\right)} \Delta u
$$


We introduce another Hilbert space

$$
\mathscr{H}_{0}=L^{2}\left(\boldsymbol{R}^{3}, \mathbb{C}^{3}, \rho_{0}\left(x_{3}\right) d x\right)
$$

with the inner product

$$
(u, v)_{\mathscr{H}_{0}}=\int_{\mathbb{R}^{3}} u \cdot \bar{v} \rho_{0}\left(x_{3}\right) d x
$$

Proposition 1.3. The operator $A_{0}$ on $\mathscr{H}_{0}$ with domain:

$$
\begin{aligned}
D\left(A_{0}\right)=\{ & u \in H^{2}\left(\mathbb{R}_{-}^{3}, \mathbb{C}^{3}\right) \oplus H^{2}\left(\mathbb{R}_{+}^{3}, C^{3}\right) \mid \\
& u \text { satisfies the interface conditions (1.21) and (1.22) } \\
& \text { in the sense of traces on } \left.x_{3}=0\right\}
\end{aligned}
$$

and action defined by

$$
A_{0} u=\mathscr{A}_{0} u, \quad u \in D\left(A_{0}\right)
$$

is a non-negative self-adjoint operator on $\mathscr{H}_{0}$.

For a proof of Proposition 1.3, see [Sh 1, Theorem 1.2].

The following notation is used to formulate our main theorem. $J$ is an identification operator from $\mathscr{H}_{0}$ into $\mathscr{H}$ defined by

$$
J f(x)=j(x) f(x) \text { for } \quad x \in \mathbb{R}^{3},
$$

where $j(x) \in C^{\infty}\left(\mathbb{R}^{3}\right)$ is a function such that $0 \leq j(x) \leq 1$, and

$$
j(x)=\left\{\begin{array}{lll}
0 & \text { for } & |x| \leq L \\
1 & \text { for } & |x| \geq L+1
\end{array}\right.
$$

Note that the operator $J$ is bounded. A real-valued function $\phi$ defined on $\mathbb{R}$ is called admissible if $\mathbb{R}$ can be divided into a finite number of subintervals $I_{1}, \cdots, I_{n}$, such that $\phi$ is continuously differentiable, locally bounded variation, and the sign of derivatives is constant in each open subinterval.

We already know that

$$
\mathscr{H}_{0}=\mathscr{H}_{0}^{a c}
$$

where $\mathscr{H}_{0}^{a c}$ is the subspace of absolutely continuous of $\mathscr{H}_{0}$ [Sh 2 , Theorem 
2.5]. The following main theorem means the existence, the completeness, and the invariance principle of the wave operators. Here the meaning of the completeness is that the wave operators $W_{ \pm}$defined below are unitary operators from $\mathscr{H}_{0}$ into the subspace of absolutely continuous of $\mathscr{H}$, and that the wave operators $W_{ \pm}^{0}$ defined below are unitary operators from the subspace of absolutely continuous of $\mathscr{H}$ into $\mathscr{H}_{0}$.

Main Theorem. If $\Omega$ is an exterior domain of which boundary $\partial \Omega$ is smooth and compact, and if the conditions (1.1)-(1.3) are satisfied, then the wave operators

$$
W_{ \pm}=W_{ \pm}\left(A, A_{0}, J\right)=\mathrm{s}-\lim _{t \rightarrow \pm \infty} e^{i t A} J e^{-i t A_{0}}
$$

and

$$
W_{ \pm}^{0}=W_{ \pm}\left(A_{0}, A, J^{*}\right)=\mathrm{s}-\lim _{t \rightarrow \pm \infty} e^{i t A_{0}} J^{*} e^{-i t A} P^{a c}
$$

exist. Here s-lim means the strong limit and $P^{a c}$ denotes the orthogonal projection of $\mathscr{H}$ onto the subspace of absolutely continuous of $\mathscr{H}$, denoted by $\mathrm{H}^{\text {ac }}$.

Moreover, the operators $W_{ \pm}: \mathscr{H}_{0} \rightarrow \mathscr{H}^{a c}$ and $W_{ \pm}^{0}: \mathscr{H}^{a c} \rightarrow \mathscr{H}_{0}$ are unitary, that is,

$$
W_{ \pm}^{*}=W_{ \pm}^{0}
$$

and the invariance principle

$$
W_{ \pm}=W_{ \pm}\left(\phi(A), \phi\left(A_{0}\right), J\right)=\mathrm{s}-\lim _{t \rightarrow \pm \infty} e^{i t \phi(A)} J e^{-i t \phi\left(A_{0}\right)}
$$

holds for any admissible function $\phi(s)$.

Every $u \in D(A)$ satisfies the interface conditions (1.5)-(1.6) and the boundary condition (1.7) in generalized sense, so the mixed problem (1.4)-(1.8) may be reformulated as the problem of finding a function $u: R \rightarrow \mathscr{H}$ such that

$$
\begin{aligned}
& \frac{d^{2} u}{d t^{2}}+A u=0 \text { for all } t \in \boldsymbol{R}, \\
& u(0)=f, \quad \frac{d u}{d t}(0)=g .
\end{aligned}
$$


Let $f$ and $g$ be real-valued functions such that $f \in \mathscr{H}, g \in D\left(A^{-\frac{1}{2}}\right)$. Then $f+i A^{-\frac{1}{2}} g \in \mathscr{H}$ and the solution $u$ belongs to $\mathscr{H}$ is given by

$$
u(t, x)=\operatorname{Re}\{v(t, x)\}, \quad v(t, \cdot)=e^{-i t A^{\frac{1}{2}}}\left(f+i A^{-\frac{1}{2}} g\right)
$$

We would like to make this main theorem have a meaning for elastic wave propagation problems. Thus we prepare the following proposition:

Proposition 1.4. The operator $A$ does not have 0 eigenvalue.

Proof. We assume that $u \in D(A)$ and $A u=0$. By integration by parts and the generalized free boundary-interface condition (1.14), we have

$$
\begin{aligned}
0=(A u, u) & =\sum_{k=1}^{3} \int_{\Omega}(\mathscr{A} u)_{k} \overline{u_{k}} \rho(x) d x \\
& =-\sum_{j, k=1}^{3} \int_{\Omega} \frac{\partial}{\partial x_{j}}\left(\lambda(x)(\nabla \cdot u) \delta_{k j}+2 \mu(x) \varepsilon_{k j}(u)\right) \overline{u_{k}} d x \\
& =\int_{\Omega}\left(\lambda(x)(\nabla \cdot u)(\nabla \cdot \bar{u})+\sum_{j . k=1}^{3} 2 \mu(x) \varepsilon_{k j}(u) \varepsilon_{k j}(\bar{u})\right) d x \\
& \geq c\|\nabla u\|^{2},
\end{aligned}
$$

where $c$ is a positive constant and $\|\cdot\|$ denotes the $L^{2}$ norm. Here the last inequality is obtained by the condition (1.1) and the following Korn's first inequality (cf. [It], [S-S], [D-L]):

$$
\sum_{k, j=1}^{3} \int_{\Omega \cap \boldsymbol{R}_{-}^{3}}\left|\varepsilon_{k j}(u)\right|^{2} d x \geq c\|\nabla u\|_{\Omega \cap \boldsymbol{R}_{-}^{3}}^{2}, \quad \sum_{k, j=1}^{3} \int_{\Omega \cap \boldsymbol{R}_{+}^{3}}\left|\varepsilon_{k j}(u)\right|^{2} d x \geq c\|\nabla u\|_{\Omega \cap \boldsymbol{R}_{+}^{3}}^{2} .
$$

It follows that the constant function $u={ }^{t}\left(c_{1}, c_{2}, c_{3}\right)$ satisfies the inequality (1.35), the interface conditions (1.5)-(1.6), and the boundary condition (1.7). However it does not belong to $L^{2}\left(\Omega, C^{3}\right)$, so that it does not belong $D(A)$. Therefore we have proved the proposition.

Proposition 1.4 was suggested by Professor Yoshihiro Shibata. 
We already know that the operator $A_{0}$ does not have 0 eigenvalue (cf. [Sh 1, Theorem 6.5]). So we deduce the following corollary from the invariance principle (1.34) in the main theorem as an admissible function $\phi(s)$ is considered as $\sqrt{s}$ :

Collorary 1.5. The wave operators

$$
W_{ \pm}\left(A^{1 / 2}, A_{0}^{1 / 2}, J\right)=\mathrm{s}-\lim _{t \rightarrow \pm \infty} e^{i t A^{1 / 2}} J e^{-i t A_{0}^{1 / 2}}
$$

and

$$
W_{ \pm}^{0}\left(A_{0}^{1 / 2}, A^{1 / 2}, J^{*}\right)=\mathrm{s}-\lim _{t \rightarrow \pm \infty} e^{i t A_{0}^{1 / 2} J^{*}} e^{-i t A^{1 / 2}} P^{a c}
$$

exixt. Moreover the operators

$$
W_{ \pm}\left(A^{1 / 2}, A_{0}^{1 / 2}, J\right): \mathscr{H}_{0} \rightarrow \mathscr{H}^{a c}
$$

and

$$
W_{ \pm}^{0}\left(A_{0}^{1 / 2}, A^{1 / 2}, J^{*}\right): \mathscr{H}^{a c} \rightarrow \mathscr{H}_{0}
$$

are unitary. Here $\mathscr{H}^{a c}$ is the subspace of absolutely continuous of $\mathscr{H}$.

\section{§. Proof of Main Theorem}

We shall prove the main theorem using an abstract operator-theoretical theorem of the Birman theory due to W. C. Lyford, which provides sufficient conditions for the existence, the completeness, and the invariance of the wave operators.

The following notation is used to formulate the abstract theorem. The scattering theory with two Hilbert spaces is concerned with a pair of self-adjoint operators $H_{0}$ and $H$ acting on separable Hilbert spaces $\mathscr{H}_{0}$ and $\mathscr{H}$, respectively, and a bounded linear operator $J$ mapping $\mathscr{H}_{0}$ into $\mathscr{H}$. We denote the spectral measure for $H_{0}$ by $\pi_{0}(\cdot)$, the subspace of absolutely continuity for $\mathscr{H}_{0}$ by $\mathscr{H}_{0}^{a c}$, the orthogonal projection of $\mathscr{H}_{0}$ onto $\mathscr{H}_{0}^{a c}$ by $P_{0}$, and $\pi_{0}^{a c}(\cdot)=P_{0} \pi_{0}(\cdot)$. Let $\pi_{0}(\cdot), \mathscr{H}^{a c}, P$, and $\pi^{a c}(\cdot)$ be defined similarly for $H$. The following classes of linear operators are needed: $B_{0}\left(\mathscr{H}_{0}, \mathscr{H}\right)$ is the class of compact linear operators from $\mathscr{H}_{0}$ to $\mathscr{H} ; B_{1}\left(\mathscr{H}_{0}, \mathscr{H}\right)$ is the class of trace-class linear operators from $\mathscr{H}_{0}$ to $\mathscr{H}$ (see $[\mathrm{Ka}]$ ).

We apply the following theorem of the Birman theory ([B-B, Theorem 
4.4]) due to W. C. Lyford ([Ly 1, Theorem 1], [Ly 2, Theorem 3.2]); moreover D. R. Yafaev [Ya, Theorem 1, p.204]. In the case where Lamé functions and a density are both perturbed, it is not known whether the limiting absorption principle for $A$ holds. Hence we need to modify Theorem 2.1 in [Sh 3] as follows (see [Wi, Appendix]):

Theorem 2.1. Suppose that

$$
J D\left(H_{0}\right) \subset D(H), \quad J^{*} D(H) \subset D\left(H_{0}\right),
$$

and we have for every bounded interval $I \subset \boldsymbol{R}$,

$$
\begin{aligned}
& \left(J^{*} J-1\right) \pi_{0}^{a c}(I) \in B_{0}\left(\mathscr{H}_{0}, \mathscr{H}_{0}\right), \\
& \left(J J^{*}-1\right) \pi^{a c}(I) \in B_{0}(\mathscr{H}, \mathscr{H}),
\end{aligned}
$$

and

$$
\left(H J-J H_{0}\right) \pi_{0}^{a c}(I) \in B_{1}\left(\mathscr{H}_{0}, \mathscr{H}\right) .
$$

Then the wave operators

$$
W_{ \pm}\left(H, H_{0}, J\right)=\mathrm{s}-\lim _{t \rightarrow \pm \infty} e^{i t H} J e^{-i t H_{0}} P_{0}
$$

and

$$
W_{ \pm}\left(H_{0}, H, J^{*}\right)=\mathrm{s}-\lim _{t \rightarrow \pm \infty} e^{i t H_{0}} J^{*} e^{-i t H} P
$$

exist. They are partial isometries and complete, and satisfy

$$
W_{ \pm}\left(H_{0}, H, J^{*}\right)=W_{ \pm}\left(H, H_{0}, J\right)^{*} \text {. }
$$

Furthermore, the invariance principle

$$
W_{ \pm}\left(H, H_{0}, J\right)=W_{ \pm}\left(\phi(H), \phi\left(H_{0}\right), J\right)
$$

holds for any admissible function $\phi(s)$.

Remark. By virtue of (1.34), we may replaced $\pi_{0}^{a c}(I)$ in (2.2) and (2.4) by $\pi_{0}(I)$. We may also omit $P_{0}$ in (2.5).

Theorem 2.1 will be applied to our problem. The self-adjoint operators 
$A$ and $A_{0}$ are replaced by $H$ and $H_{0}$, respectively. Other notations can be used as they are.

We verify the sufficient conditions (2.1)-(2.4). The condition (2.1) becomes as follows:

$$
J D\left(A_{0}\right) \subset D(A), \quad J^{*} D(A) \subset D\left(A_{0}\right)
$$

where $D(A)$ and $D\left(A_{0}\right)$ are defined by (1.17) and (1.26), respectively, and $J^{*}$ is the bounded operator from $\mathscr{H}$ into $\mathscr{H}_{0}$ defined by

$$
J^{*} f(x)=\left\{\begin{array}{lll}
j(x) f(x) & \text { for } & x \in \Omega, \\
0 & \text { for } & x \in \boldsymbol{R}^{3} \backslash \Omega .
\end{array}\right.
$$

The validity of these conditions is obvious from (1.28), (1.29), and (2.10), because $j(x)$ vanishes in a neighborhood of $\boldsymbol{R}^{3} \backslash \Omega$.

To verify the sufficient condition (2.4), it is necessary to calculate the operator $A J-J A_{0}$. From the condition (2.9), we have for $u \in D\left(A_{0}\right)$

$$
\begin{gathered}
A J u=\mathscr{A}\{j(x) u(x)\}=(-1) \times \frac{1}{\rho(\cdot)} \times \\
\frac{\partial}{\partial x_{1}}\{\lambda(\cdot)+2 \mu(\cdot)\} \frac{\partial}{\partial x_{1}}+\frac{\partial}{\partial x_{2}} \mu(\cdot) \frac{\partial}{\partial x_{2}}+\frac{\partial}{\partial x_{3}} \mu(\cdot) \frac{\partial}{\partial x_{3}} \\
\frac{\partial}{\partial x_{1}} \mu(\cdot) \frac{\partial}{\partial x_{2}}+\frac{\partial}{\partial x_{2}} \lambda(\cdot) \frac{\partial}{\partial x_{1}} \\
\frac{\partial}{\partial x_{1}} \mu(\cdot) \frac{\partial}{\partial x_{3}}+\frac{\partial}{\partial x_{3}} \lambda(\cdot) \frac{\partial}{\partial x_{1}} \\
\frac{\partial}{\partial x_{1}} \lambda(\cdot) \frac{\partial}{\partial x_{2}}+\frac{\partial}{\partial x_{2}} \mu(\cdot) \frac{\partial}{\partial x_{1}} \\
\frac{\partial}{\partial x_{1}} \mu(\cdot) \frac{\partial}{\partial x_{1}}+\frac{\partial}{\partial x_{2}}\{\lambda(\cdot)+2 \mu(\cdot)\} \frac{\partial}{\partial x_{2}}+\frac{\partial}{\partial x_{3}} \mu(\cdot) \frac{\partial}{\partial x_{3}} \\
\frac{\partial}{\partial x_{2}} \mu(\cdot) \frac{\partial}{\partial x_{3}}+\frac{\partial}{\partial x_{3}} \lambda(\cdot) \frac{\partial}{\partial x_{2}}
\end{gathered}
$$




$$
\left.\begin{array}{c}
\frac{\partial}{\partial x_{1}} \lambda(\cdot) \frac{\partial}{\partial x_{3}}+\frac{\partial}{\partial x_{3}} \mu(\cdot) \frac{\partial}{\partial x_{1}} \\
\frac{\partial}{\partial x_{2}} \lambda(\cdot) \frac{\partial}{\partial x_{3}}+\frac{\partial}{\partial x_{3}} \mu(\cdot) \frac{\partial}{\partial x_{2}} \\
\frac{\partial}{\partial x_{1}} \mu(\cdot) \frac{\partial}{\partial x_{1}}+\frac{\partial}{\partial x_{2}} \mu(\cdot) \frac{\partial}{\partial x_{2}}+\frac{\partial}{\partial x_{3}}\{\lambda(\cdot)+2 \mu(\cdot)\} \frac{\partial}{\partial x_{3}}
\end{array}\right)
$$

and

$$
J A_{0} u(x)=j(x)\left\{\mathscr{A}_{0} u(x)\right\}=j(x) \times(-1) \times \frac{1}{\rho_{0}}
$$

$$
\left(\begin{array}{cc}
\left(\lambda_{0}+2 \mu_{0}\right) \frac{\partial^{2}}{\partial x_{1}^{2}}+\mu_{0}\left(\frac{\partial^{2}}{\partial x_{2}^{2}}+\frac{\partial^{2}}{\partial x_{3}^{2}}\right) & \left(\lambda_{0}+\mu_{0}\right) \frac{\partial^{2}}{\partial x_{1} \partial x_{2}} \\
\left(\lambda_{0}+\mu_{0}\right) \frac{\partial^{2}}{\partial x_{1} \partial x_{2}} & \left(\lambda_{0}+2 \mu_{0}\right) \frac{\partial^{2}}{\partial x_{2}^{2}}+\mu_{0}( \\
\left(\lambda_{0}+\mu_{0}\right) \frac{\partial^{2}}{\partial x_{1} \partial x_{3}} & \left(\lambda_{0}+\mu_{0}\right) \frac{\partial^{2}}{\partial x_{2} \partial x_{3}}
\end{array}\right.
$$

$$
\left.\begin{array}{c}
\left(\lambda_{0}+\mu_{0}\right) \frac{\partial^{2}}{\partial x_{1} \partial x_{3}} \\
\left(\lambda_{0}+\mu_{0}\right) \frac{\partial^{2}}{\partial x_{2} \partial x_{3}} \\
\left.\lambda_{0}+2 \mu_{0}\right) \frac{\partial^{2}}{\partial x_{3}^{2}}+\mu_{0}\left(\frac{\partial^{2}}{\partial x_{1}^{2}}+\frac{\partial^{2}}{\partial x_{2}^{2}}\right)
\end{array}\right)\left(\begin{array}{l}
u_{1}(x) \\
u_{2}(x) \\
u_{3}(x)
\end{array}\right),
$$

where $\lambda_{0}=\lambda_{0}\left(x_{3}\right), \mu_{0}=\mu_{0}\left(x_{3}\right)$ and $\rho_{0}=\rho_{0}\left(x_{3}\right)$. For simplicity we only consider the $(3,1)$-component with respect to $\lambda(x)$ and $\lambda$ of $A J-J A_{0}$ and denote it by $\left(A J-J A_{0}\right)_{(3,1) \lambda}$. Other components can be treated in a similar way. By (2.11) and (2.12), we have 
(2.13)

$$
\begin{aligned}
& \left(A J-J A_{0}\right)_{(3,1) \lambda} u_{1}(x) \\
= & (-1) \times\left[\frac{1}{\rho(x)} \frac{\partial}{\partial x_{3}} \lambda(x) \frac{\partial}{\partial x_{1}}\left(j(x) u_{1}(x)\right)-\frac{1}{\rho_{0}\left(x_{3}\right)} j(x) \lambda_{0}\left(x_{3}\right) \frac{\partial^{2}}{\partial x_{3} \partial x_{1}} u_{1}(x)\right] \\
= & (-1) \times\left[j(x)\left(\frac{\lambda(x)}{\rho(x)}-\frac{\lambda_{0}\left(x_{3}\right)}{\rho_{0}\left(x_{3}\right)}\right) \frac{\partial^{2}}{\partial x_{3} \partial x_{1}} u_{1}(x)\right. \\
& +\frac{1}{\rho(x)} \frac{\partial}{\partial x_{3}} \lambda(x) \frac{\partial}{\partial x_{1}} j(x) u_{1}(x)+\frac{\lambda(x)}{\rho(x)} \frac{\partial^{2}}{\partial x_{3} \partial x_{1}} j(x) u_{1}(x) \\
& +\frac{\lambda(x)}{\rho(x)} \frac{\partial}{\partial x_{1}} j(x) \frac{\partial}{\partial x_{3}} u_{1}(x)+\frac{1}{\rho(x)} \frac{\partial}{\partial x_{3}} \lambda(x) j(x) \frac{\partial}{\partial x_{1}} u_{1}(x) \\
& \left.+\frac{\lambda(x)}{\rho(x)} \frac{\partial}{\partial x_{3}} j(x) \frac{\partial}{\partial x_{1}} u_{1}(x)\right]
\end{aligned}
$$

for all $u_{1}(x)$ such that $u(x)={ }^{t}\left(u_{1}(x), u_{2}(x), u_{3}(x)\right) \in D\left(A_{0}\right)$.

The spectral family $\pi_{0}(s)$ has the following expression:

$$
\pi_{0}(s) f(x)= \begin{cases}\sum_{j \in M}\left(\int_{|\eta| \leq \sqrt{s} / c_{j}} \psi_{1 j}^{ \pm}(x, \eta) \hat{f}_{1 j}^{ \pm}(\eta) d \eta\right. \\ \left.\quad+\int_{-\infty}^{+\infty} \int_{\left|\eta^{\prime}\right| \leq \sqrt{s} / c_{s t}} \psi_{1 j}^{S t}(x, \eta) \hat{f}_{1 j}^{S t}(\eta) d \eta^{\prime} d \xi\right) \\ +\sum_{k \in N} \int_{|\eta| \leq \sqrt{s} / c_{k}} \psi \frac{ \pm}{2 k}(x, \eta) \hat{f}_{2 k}^{ \pm}(\eta) d \eta, & s \geq 0, \\ 0, & s<0 .\end{cases}
$$

Here $\eta=\left(\eta_{1}, \eta_{2}, \xi\right)=\left(\eta^{\prime}, \xi\right)$ are the dual variables of $\left(x_{1}, x_{2}, x_{3}\right)$. The $c_{j}^{2}|\eta|^{2}$ $\left(j \in M=\left\{s_{1}, p_{1}, s_{2}, p_{2}\right\}\right)$ and $c_{k}^{2}|\eta|^{2}\left(k \in N=\left\{s_{1}, s_{2}\right\}\right)$ are the roots of characteristic equation of elastic equation (1.24). The $c_{S t}\left|\eta^{\prime}\right|$ is the square root of the zero of Lopatinski determinant, and $c_{S t}$ is the speed of the interface wave which is called Stonely wave. The $\psi_{1 j}^{ \pm}(x, \eta), \psi_{1 j}^{S t}(x, \eta)(j \in M)$ and $\psi_{2 k}^{ \pm}(x, \eta)(k \in N)$ are generalized eigenfunctions for $A_{0}$. Moreover $\left\{\hat{f}_{1 j}^{ \pm}, \hat{f}_{j}^{S t}, \hat{f}_{2 k}^{ \pm}\right\}_{j \in M, k \in N}$ are the Fourier transforms of $f \in \mathscr{H}$ with respect to the generalized eigenfunctions $\left\{\hat{\psi}_{1 j}^{ \pm}, \hat{\psi}_{j}^{S t}, \hat{\psi}_{2 k}^{ \pm}\right\}_{j \in M . k \in N}$, respectively (cf. [Sh 3, (A.16)]).

It follows that

$$
\pi_{0}(I) u(x)=\sum_{j \in M}\left(\int_{c_{j}^{2}|\eta|^{2} \in I} \psi_{1 j}^{ \pm}(x, \eta) \hat{u}_{1 j}^{ \pm}(\eta) d \eta+\int_{c_{s_{t}}^{2}\left|\eta^{\prime}\right|^{2} \in I} \psi_{1 j}^{S t}(x, \eta) \hat{u}_{1 j}^{S t}(\eta) d \eta\right)
$$




$$
+\sum_{k \in N} \int_{c_{k}^{2}|\eta|^{2} \in I} \psi \frac{ \pm}{2 k}(x, \eta) \hat{u}_{2 k}^{ \pm}(\eta) d \eta,
$$

where $I$ is an arbitrary bounded interval. Combining (2.13) and (2.15) gives that

$$
\begin{aligned}
& \left(A J-J A_{0}\right)_{(3,1) \lambda}\left(\pi_{0}(I) u(x)\right)_{1} \\
& =(-1) \times\left[j(x)\left(\frac{\lambda(x)}{\rho(x)}-\frac{\lambda_{0}\left(x_{3}\right)}{\rho_{0}\left(x_{3}\right)}\right) \frac{\partial^{2}}{\partial x_{3} \partial x_{1}}\left(\pi_{0}(I) u(x)\right)_{1}\right. \\
& \quad+\frac{1}{\rho(x)} \frac{\partial}{\partial x_{3}} \lambda(x) \frac{\partial}{\partial x_{1}} j(x)\left(\pi_{0}(I) u(x)\right)_{1}+\frac{\lambda(x)}{\rho(x)} \frac{\partial^{2}}{\partial x_{3} \partial x_{1}} j(x)\left(\pi_{0}(I) u(x)\right)_{1} \\
& \quad+\frac{\lambda(x)}{\rho(x)} \frac{\partial}{\partial x_{1}} j(x) \frac{\partial}{\partial x_{3}}\left(\pi_{0}(I) u(x)\right)_{1}+\frac{1}{\rho(x)} \frac{\partial}{\partial x_{3}} \lambda(x) j(x) \frac{\partial}{\partial x_{1}}\left(\pi_{0}(I) u(x)\right)_{1} \\
& \left.\quad+\frac{\lambda(x)}{\rho(x)} \frac{\partial}{\partial x_{3}} j(x) \frac{\partial}{\partial x_{1}}\left(\pi_{0}(I) u(x)\right)_{1}\right]
\end{aligned}
$$

where $\left(\pi_{0}(I) u(x)\right)_{1}$ denotes the first component of the column vector $\pi_{0}(I) u(x)$.

The expression (2.16) implies that

$$
\left(A J-J A_{0}\right) \pi_{0}(I)=\sum_{j \in M} T_{1 j}^{ \pm} \Phi_{1 j}^{ \pm}+\sum_{j \in M} T_{1 j}^{S t} \Phi_{1 j}^{S t}+\sum_{k \in N} T_{2 k}^{ \pm} \Phi_{2 k}^{ \pm}
$$

where $\Phi_{1 j}^{ \pm}, \Phi_{1 j}^{S t}$ and $\Phi_{2 k}^{ \pm}$are the generalized Fourier transforms (Cf. [Sh 1], [Sh 3, Appendix])

$$
\begin{aligned}
\Phi_{1 j}^{ \pm}, \Phi_{2 k}^{ \pm}: \mathscr{H}_{0} & \rightarrow L^{2}\left(\boldsymbol{R}_{+}^{3}, C^{3}\right) \text { for } \xi>0, \rightarrow L^{2}\left(\boldsymbol{R}_{-}^{3}, C^{3}\right) \text { for } \xi<0 \\
\Phi_{1 j}^{S t}: \mathscr{H}_{0} & \rightarrow L^{2}\left(\boldsymbol{R}^{3}, C^{3}\right)
\end{aligned}
$$

and the integral operators $T_{1 j}^{ \pm}, T_{2 k}^{ \pm}: L^{2}\left(\boldsymbol{R}_{ \pm}^{3}, C^{3}\right) \rightarrow \mathscr{H}$ and $T_{1 j}^{S t}: L^{2}\left(\boldsymbol{R}^{3}, C^{3}\right) \rightarrow \mathscr{H}$ are defined respectively by the formulas

$$
\begin{aligned}
& T_{1 j}^{ \pm} f(x)=\int_{K_{1_{j}}^{ \pm}} \tau_{1 j}^{ \pm}(x, \eta) f(\eta) d \eta, \quad x \in \Omega \\
& T_{1 j}^{S t} f(x)=\int_{K_{1 j}^{S t}} \tau_{1 j}^{S t}(x, \eta) f(\eta) d \eta, \quad x \in \Omega
\end{aligned}
$$




$$
T_{2 k}^{ \pm} f(x)=\int_{K_{2 k}^{ \pm}} \tau \frac{ \pm}{2 k}(x, \eta) f(\eta) d \eta, \quad x \in \Omega,
$$

where $j \in M=\left\{p_{1}, s_{1}, p_{2}, s_{2}\right\}$ and $k \in N=\left\{s_{1}, s_{2}\right\}$. As to (2.19) see [Sh 2, (3.5)]. Here

$$
\begin{aligned}
& K_{1 j}^{ \pm}=\left\{\left.\eta \in \boldsymbol{R}_{ \pm}^{3}\left|c_{j}^{2}\right| \eta\right|^{2} \in I\right\} ; \\
& K_{1 j}^{S t}=\left\{\left.\eta \in \boldsymbol{R}^{3}\left|c_{S t}^{2}\right| \eta^{\prime}\right|^{2} \in I, \quad \xi \in \boldsymbol{R}\right\} ; \\
& K_{2 k}^{ \pm}=\left\{\left.\eta \in \boldsymbol{R}_{ \pm}^{3}\left|c_{k}^{2}\right| \eta\right|^{2} \in I\right\} .
\end{aligned}
$$

The (3,1)-component of the integral kernel $\tau_{1 p_{1}}^{+}(x, \eta)$ is the sum of the following term (2.24) and similar terms (cf. [Sh 1, (4.9)]), and the other components have similar expressions:

$$
\begin{aligned}
- & (2 \pi)^{-3 / 2} e^{i\left(x_{1} \eta_{1}+x_{2} \eta_{2}\right)} \frac{\eta_{1}}{\left|\eta^{\prime}\right|\left|\eta^{2}\right|} \frac{1}{\rho_{1}} \\
\times & {\left[\left(j(x)\left(\frac{\lambda_{1}(x)}{\rho_{1}(x)}-\frac{\lambda_{1}}{\rho_{1}}\right) i \eta_{1}+\frac{\lambda_{1}(x)}{\rho_{1}(x)} \frac{\partial}{\partial x_{1}} j(x)\right)\right.} \\
\times & i\left(\frac{\Delta_{p_{1}}^{1}\left(\eta^{\prime}, \lambda_{p_{1}}\right)}{\Delta\left(\eta^{\prime}, \lambda_{p_{1}}\right)} e^{-i \xi x_{3} \xi\left|\eta^{\prime}\right|^{2}}\right. \\
& +\frac{\Delta_{p_{1}}^{2}\left(\eta^{\prime}, \lambda_{p_{1}}\right)}{\Delta\left(\eta^{\prime}, \lambda_{p_{1}}\right)} e^{-i \xi \xi_{s_{1}}\left(\eta^{\prime}, \lambda_{p_{1}}\right) x_{3}} \xi_{s_{1}}^{2}\left(\eta^{\prime}, \lambda_{p_{1}}\right)\left|\eta^{\prime}\right|+e^{\left.i \xi x_{3} \xi\left|\eta^{\prime}\right|^{2}\right)} \\
& -\left(\frac{1}{\rho_{1}(x)} \frac{\partial}{\partial x_{3}} \lambda_{1}(x) \frac{\partial}{\partial x_{1}} j(x)+\frac{\lambda_{1}(x)}{\rho_{1}(x)} \frac{\partial^{2}}{\partial x_{3} \partial x_{1}} j(x)\right. \\
& \left.+\frac{1}{\rho_{1}(x)} \frac{\partial}{\partial x_{3}} \lambda_{1}(x) j(x) i \eta_{1}+\frac{\lambda_{1}(x)}{\rho_{1}(x)} \frac{\partial}{\partial x_{3}} j(x) i \eta_{1}\right) \\
\times & \left(-\frac{\Delta_{p_{1}}^{1}\left(\eta^{\prime}, \lambda_{p_{1}}\right)}{\Delta\left(\eta^{\prime}, \lambda_{p_{1}}\right)} e^{-i \xi \xi_{3}\left|\eta^{\prime}\right|^{2}}\right. \\
& -\frac{\Delta_{p_{1}}^{2}\left(\eta^{\prime}, \lambda_{p_{1}}\right)}{\Delta\left(\eta^{\prime}, \lambda_{p_{1}}\right)} e^{\left.\left.-i \xi_{s_{1}}\left(\eta^{\prime} \cdot \lambda_{p_{1}}\right) x_{3} \xi_{s_{1}}\left(\eta^{\prime}, \lambda_{p_{1}}\right)\left|\eta^{\prime}\right|+e^{i \xi x_{3}}\left|\eta^{\prime}\right|^{2}\right)\right]}
\end{aligned}
$$




$$
\text { for } x \in \Omega \cap \boldsymbol{R}_{-}^{3}, \eta \in K_{1 p_{1}}^{+} \text {, }
$$

where

$$
\xi_{s_{1}}\left(\eta^{\prime}, \lambda_{p_{1}}\right)= \pm \sqrt{\frac{c_{p_{1}}^{2}|\eta|^{2}}{c_{s_{1}}^{2}}-\left|\eta^{\prime}\right|^{2}},
$$

and $\Delta_{p_{1}}^{l}\left(\eta^{\prime}, \lambda_{p_{1}}\right)(l=1,2,3,4)$ denote the determinants respectively obtained from $\Delta\left(\eta^{\prime}, \lambda_{p_{1}}\right)=$

$$
\left|\begin{array}{cccc}
\left|\eta^{\prime}\right| & \xi_{s_{1}} & -\left|\eta^{\prime}\right| & \xi_{s_{2}} \\
-\xi & \left|\eta^{\prime}\right| & -\xi_{p_{2}} & -\left|\eta^{\prime}\right| \\
-2 \rho_{1} c_{s_{1}}^{2} \xi\left|\eta^{\prime}\right| & -\rho_{1} c_{s_{1}}^{2}\left(\xi_{s_{1}}^{2}-\left|\eta^{\prime}\right|^{2}\right) & -2 \rho_{2} c_{s_{2}}^{2} \xi_{p_{2}}\left|\eta^{\prime}\right| & \rho_{2} c_{s_{2}}^{2}\left(\xi_{s_{2}}^{2}-\left|\eta^{\prime}\right|^{2}\right) \\
\rho_{1} c_{s_{1}}^{2}\left(\xi_{s_{1}}^{2}-\left|\eta^{\prime}\right|^{2}\right) & -2 \rho_{1} c_{s_{1}}^{2} \xi_{s_{1}}\left|\eta^{\prime}\right| & -\rho_{2} c_{s_{2}}^{2}\left(\xi_{s_{2}}^{2}-\left|\eta^{\prime}\right|^{2}\right) & -2 \rho_{2} c_{s_{2}}^{2} \xi_{s_{2}}\left|\eta^{\prime}\right|
\end{array}\right|
$$

by replacing the $l$ th column by (cf. [Sh 1, (2.22)])

$$
{ }^{t}\left(\left|\eta^{\prime}\right|, \quad \xi, \quad 2 \rho_{1} c_{s_{1}}^{2} \xi\left|\eta^{\prime}\right|, \quad \rho_{1} c_{s_{1}}^{2}\left(\xi_{s_{1}}^{2}\left(\eta^{\prime}, \lambda_{p_{1}}\right)-\left|\eta^{\prime}\right|^{2}\right)\right)
$$

Here

$$
\begin{aligned}
& \xi_{p_{2}}=\xi_{p_{2}}\left(\eta^{\prime}, \lambda_{p_{1}}\right)= \begin{cases} \pm \sqrt{\frac{c_{p_{1}}^{2}\left(\left|\eta^{\prime}\right|^{2}+\xi^{2}\right)}{c_{p_{2}}^{2}}-\left|\eta^{\prime}\right|^{2}} & c_{p_{1}}^{2}|\eta|^{2}>c_{p_{2}}^{2}\left|\eta^{\prime}\right|^{2}, \\
i \sqrt{\left|\eta^{\prime}\right|^{2}-\frac{c_{p_{1}}^{2}\left(\left|\eta^{\prime}\right|^{2}+\xi^{2}\right)}{c_{p_{2}}^{2}}} & c_{p_{1}}^{2}|\eta|^{2}<c_{p_{2}}^{2}\left|\eta^{\prime}\right|^{2},\end{cases} \\
& \xi_{s_{2}}=\xi_{s_{2}}\left(\eta^{\prime}, \lambda_{p_{1}}\right)= \begin{cases} \pm \sqrt{\frac{c_{p_{1}}^{2}\left(\left|\eta^{\prime}\right|^{2}+\xi^{2}\right)}{c_{s_{2}}^{2}}-\left|\eta^{\prime}\right|^{2}} & c_{p_{1}}^{2}|\eta|^{2}>c_{s_{2}}^{2}\left|\eta^{\prime}\right|^{2}, \\
i \sqrt{\left|\eta^{\prime}\right|^{2}-\frac{c_{p_{1}}^{2}\left(\left|\eta^{\prime}\right|^{2}+\xi^{2}\right)}{c_{p_{2}}^{2}}} & c_{p_{1}}^{2}|\eta|^{2}<c_{s_{2}}^{2}\left|\eta^{\prime}\right|^{2} .\end{cases}
\end{aligned}
$$

The components of the other integral kernels $\tau_{1 p_{1}}^{-}, \tau_{1 j}^{ \pm}\left(j \in\left\{s_{1}, p_{2}, s_{2}\right\}\right), \tau_{1 j}^{S t}$ $(j \in M)$ and $\tau_{2 k}^{ \pm}(k \in N)$ also have similar expressions.

Criteria in order that the integral operators of the form $(2.18)-(2.20)$ be of trace-class are given by W. F. Stinespring ([St, Theorem 2]). Here we shall use a special case of the criteria due to Wilcox ([Wi, Theorem A.2]). 
Theorem 2.2. Assume that $\operatorname{supp} \tau_{1 j}^{ \pm}(\cdot, \eta)\left(\eta \in K_{1 j}^{ \pm}\right)$, $\operatorname{supp} \tau_{1 j}^{S t}(\cdot, \eta)\left(\eta \in K_{1 j}^{S t}\right)$, and $\operatorname{supp} \tau_{2 k}^{ \pm}(\cdot, \eta)\left(\eta \in K_{2 k}^{ \pm}\right)$are compact and that $\tau_{1 j}^{ \pm}(x, \cdot) \in H^{m}\left(\boldsymbol{R}_{ \pm}^{3}, \mathscr{H}\right)\left(x \in \Omega \cap \boldsymbol{R}_{ \pm}^{3}\right)$, $\tau_{1 j}^{S t}(x, \cdot) \in H^{m}\left(\boldsymbol{R}^{3}, \mathscr{H}\right)\left(x \in \Omega \cap \boldsymbol{R}_{ \pm}^{3}\right)$, and $\tau_{2 k}^{ \pm}(x, \cdot) \in H^{m}\left(\boldsymbol{R}_{ \pm}^{3}, \mathscr{H}\right)\left(x \in \Omega \cap \boldsymbol{R}_{ \pm}^{3}\right)$ for some $m>3 / 2$. Then the operators $T_{1 j}^{ \pm}, T_{1 j}^{S t}, T_{2 k}^{ \pm}$define operators in $B_{1}\left(L^{2}\left(\boldsymbol{R}_{ \pm}^{3}, C^{3}\right), \mathscr{H}\right)$, $B_{1}\left(L^{2}\left(\boldsymbol{R}^{3}, C^{3}\right), \mathscr{H}\right)$, and $B_{1}\left(L^{2}\left(\boldsymbol{R}_{ \pm}^{3}, C^{3}\right), \mathscr{H}\right)$, respectively.

To apply Theorem 2.2 to the operator $T_{1 p_{1}}^{ \pm}$, note that the integral kernel $\tau_{1 p_{1}}^{+}(x, \eta)$ can be extended to the outside of $\eta \in K_{1 p_{1}}^{+}$. We multiply the term (2.24), which is the typical term of the $(3,1)$-components of the integral kernel $\tau_{1 p_{1}}^{+}(x, \eta)$, by $\phi(\eta)$, where $x \in \Omega \cap \boldsymbol{R}_{-}^{3}, \phi \in \mathscr{D}\left(\boldsymbol{R}^{3}\right)$ and $\phi(\eta)=1$ for all $\eta \in K_{1 p_{1}}^{+}$. The other components of the integral kernel $\tau_{1 p_{1}}^{+}(x, \eta)$ have similar expressions. It is clear that $\tau_{1 p_{1}}^{+}(x, \cdot) \in H^{2}\left(\boldsymbol{R}_{+}^{3}, \mathscr{H}\right)$ and that

$$
\operatorname{supp} \tau_{1 p_{1}}^{ \pm}(\cdot, \eta) \subset \Omega \cap \boldsymbol{R}_{-}^{3} \cap\{x|| x \mid \leq L+1\} \text { for all } \eta \in \boldsymbol{R}_{+}^{3},
$$

because of the assumptions (1.1)-(1.3). The other integral kernels $\tau_{1 p_{1}}^{-}, \tau_{1 j}^{ \pm}$ $\left(j \in\left\{s_{1}, p_{2}, s_{2}\right\}\right), \tau_{1 j}^{S t}(j \in M)$ and $\tau_{2 k}^{ \pm}(k \in N)$ have similar results. Thus Theorem 2.2 implies that

$$
\left(A J-J A_{0}\right) \pi_{0}(I) \in B_{1}\left(\mathscr{H}_{0}, \mathscr{H}\right)
$$

because $T_{1 j}^{ \pm}, T_{2 k}^{ \pm} \in B_{1}\left(L^{2}\left(\boldsymbol{R}_{ \pm}^{3}, C^{3}\right), \mathscr{H}\right), \Phi_{1 j}^{ \pm}, \Phi_{2 k}^{ \pm} \in B\left(\mathscr{H}_{0}, L^{2}\left(\boldsymbol{R}_{ \pm}^{3}, C^{3}\right)\right)$, and $T_{1 j}^{S t}$ $\in B_{1}\left(L^{2}\left(\boldsymbol{R}^{3}, C^{3}\right), \mathscr{H}\right), \Phi_{1 j}^{S t} \in B\left(\mathscr{H}_{0}, L^{2}\left(\boldsymbol{R}^{3}, C^{3}\right)\right)$ for (2.17). This completes the verification of the condition (2.4).

Finally we verify the condition (2.3) as in the Appendix of [Wi, pp.175-176].

Let $\left\{u_{m}\right\}$ be a bounded sequence in $\mathscr{H}$. We shall show that

$$
\left(j^{2}(x)-1\right) \pi^{a c}(I) u_{m}(x)
$$

has a subsequence which converges in $\mathscr{H}$. Since

$$
\operatorname{supp}\left(j^{2}(x)-1\right) \subset\{x|| x \mid \leq L+1\},
$$

it is enough to show that $\left\{\pi^{a c}(I) u_{m}\right\}$ has a subsequence which converges in $L^{2}\left(\Omega \cap\{x|| x \mid \leq L+1\}, C^{3}, \rho(x)\right)$. By Korn's inequality (1.36), we have

$$
\left\|\nabla\left(\pi^{a c}(I) u_{m}\right)\right\|^{2} \leq C .
$$

Thus $\left\{\pi^{a c}(I) u_{m}\right\}$ has a convergent subsequence in $L^{2}\left(\Omega \cap\{x|| x \mid \leq L+1\}, C^{3}\right.$, $\rho(x))$ by means of Rellich's local compactness theorem. 
The condition (2.2) is verified just same as in the condition (2.3). This completes the proof of Main Theorem.

\section{Acknowledgement}

I would like to express my sincere thanks to Professors Shige Toshi Kuroda and Mutsuhide Matsumura for their valuable advices. I am also grateful to the referee for his helpful criticisms of the manuscript.

\section{References}

[B-B] Belopol'skii, A. L. and Birman, M. S.., The existence of wave operators in scattering theory for pairs of spaces, Izv. Acad. Nauk SSSR Ser. Mat., 32 (1968), 1117-1130.

[D-G] Dermenjian, Y. and Guillot, J. C., Scattering of elastic waves in a perturbed isotropic half space with a free boundary. The limiting absorption principle, Math. Meth. Appl. Sci., 10 (1988), 87-124.

[D-L] Duvaut, G. and Lions, J. L., Inequalities in Mechanics and Physics, Springer-Verlag, Berlin Heidelberg New York, 1976.

[It] Ito, H., Extended Korn's inequalities and the associated best possible constants, J. Elasticity, 24 (1990), 43-78.

[Ka] Kato, T., Perturbation Theory for Linear Operators, Springer-Verlag, Berlin Heidelberg New York, 1966.

[Ly 1] Lyford, W. C., A two Hilbert space scattering theorem, Math. Ann., 217 (1975), 257-261.

[Ly 2] Spectral analysis of the Laplacian in domains with cylinders, Math. Ann., 218 (1975), 229-251.

[Mi] Mizohata, S., The Theory of Partial Differential Equations, Cambridge University Press, London, 1973.

[Sh 1] Shimizu, S., Eigenfunction expansions for elastic wave propagation problems in stratified media $R^{3}$, Tsukuba J. Math., 18 (1994), 283-350.

[Sh 2] - The limiting absorption principle for elastic wave propagation problems in perturbed stratified media $R^{3}$, Math. Meth. Appl. Sci., 19 (1996), 187-215.

[Sh 3] - Scattering Theory for elastic wave propagation problems in perturbed stratified media $R^{3}$, Funkcial. Ekvac., 40 (1997), 57-77.

[S-S] Shibata, Y. and Soga, H., Scattering theory for the elastic wave equation, Publ. RIMS, Kyoto Univ., 25 (1989), 861-887.

[St] Stinespring, W. F., A sufficient condition for an integral operator to have a trace, J. Reine Angew. Math., 200 (1958), 200-207.

[Wi] Wilcox, C. H., Scattering Theory for the d'Alembert Equation in Exterior Domains; Springer Lecture Notes in Math. 442, Springer-Verlag, Berlin, Heidelberg, New York, 1975.

[Ya] Yafaev, D. R., Mathematical Scattering Theory, A.M.S. Transl. 105, Providence, Rhode Island, 1992. 